\title{
Culture and therapist self-disclosure
}

\author{
Peter Phiri ${ }^{1,2 *}$, Shanaya Rathod ${ }^{1}$, Mary Gobbi ${ }^{2}$, Hannah Carr $^{1}$ and David Kingdon ${ }^{1,2}$ \\ ${ }^{1}$ Southern Health NHS Foundation Trust, UK and ${ }^{2}$ University of Southampton, Southampton, UK \\ *Corresponding author. Email: peter.phiri@nhs.net
}

(Received 22 March 2018; revised 05 February 2019; accepted 15 February 2019)

\begin{abstract}
Cognitive behaviour therapy (CBT) as a treatment for schizophrenia and psychotic-related disorders has been shown to have significantly greater drop-out rates in clients of black and minority ethnic (BME) groups. This has resulted in poor outcomes in treatments. Our recent qualitative study thus aimed to develop culturally sensitive CBT for BME clients. The study consisted of individual in-depth 1:1 interviews with patients with a diagnosis of schizophrenia, schizo-affective, delusional disorders or psychosis $(n=15)$ and focus groups with lay members $(n=52)$, CBT therapists $(n=22)$ and mental health practitioners $(n=25)$ on a data set of 114 participants. Several themes emerged relating to therapist awareness on culturally derived behaviours, beliefs and attitudes that can influence client response and participation in therapy. The current paper aims to explore one of these themes in greater detail, i.e. client-initiated therapist self-disclosure (TSD). Using thematic analysis, the paper highlights key elements of TSD and how this could impact on therapist's reactions towards TSD, the therapeutic alliance and ultimately, the outcomes of therapy. The findings appear to show that TSD has significant relevance in psychological practice today. Some BME client groups appear to test therapists through initiating TSD. It is not the content of TSD they are testing per se, but how the therapist responds. Consequently, this requires therapists' cognisance and sensitive responses in a manner that will nurture trust and promote rapport. Further investigation in this area is suggested with a recommendation for guidelines to be created for clinicians and training.
\end{abstract}

\section{Key learning aims}

(1) To develop a dialogue and practice with confidence when addressing issues of self-disclosure with diverse populations.

(2) To appreciate the impact therapist self-disclosure has in early stages of engagement, in particular when working with patients from BME communities.

(3) To understand the impact and role of self-disclosure as initiated by patients.

(4) To increase therapist awareness on cultural differences in self-disclosure and develop ways to address this in therapy.

(5) To challenge therapists to adapt psychological therapies to diverse cultures and be cognisant that 'one size does not fit all'.

Keywords: cognitive behaviour therapy; culture; ethnic minority; psychotherapy; self-disclosure

\section{Introduction}

There has been an increase in the population of black and minority ethnic groups (BME) within the UK. In 2011 the figure had risen to 14\% compared with that of $5.9 \%$ in 1991 (Office for National Statistics, 2011). This increase was largely due to immigration from Eastern Europe and from migrants from Commonwealth countries. 
The prevalence of mental illness in the UK is estimated at one in four adults each year. However a pooled incidence of schizophrenia has been estimated at 15 per 100,000 people. In relation to mental health, there is significant and independent variation of incidence of schizophrenia and other psychoses in terms of ethnicity, with all psychoses reported to be more common in BME groups (crude incidence rate ratio, 3.6; Kirkbride et al., 2006). Cantor-Graae and Selten's (2005) meta-analysis of 18 studies demonstrated a significant increased risk of schizophrenia in migrant groups from the Global South, with significant variation of risk by both host countries and the countries of origin. The literature shows rates of schizophrenia to be two to 14 times greater for African-Caribbean than for whites in the UK (Fearon and Morgan, 2006; King et al., 2005). Compared with their 'native' white counterparts, patients from BME groups with schizophrenia are also likely to be misunderstood and misdiagnosed and more likely to be treated with drugs and electroconvulsive therapy (ECT) rather than psychotherapy (Fernando, 1988; Fearon et al., 2006) and are less likely to have their social and psychological needs addressed within the care planning process (Bhui, 2002) leading to dissatisfaction with services (Bhugra, 1997; Bhui, 1997). In 2011, the sixth and final 'Count Me In' census was published by the Office for National Statistics; this reported a continued increase in mental health admissions and detention rates in BME groups. Of note were the significantly higher rates of detention for Black African and Chinese; in contrast, the White British group had lower than averages rates of admission. However, it has been shown that in Global South countries the prevalence of schizophrenia is lower than in western countries. In North America for instance, the statistics for African Americans were similar to their British counterparts (Jackson et al., 2007).

Similarly, the Asian groups had average rates of admission (Healthcare Commission, 2008). This disparity when compared with African Caribbeans is attributed by some scholars to supportive family structures within the Asian communities (Raleigh, 2000). Kirkbride et al. (2012) conducted a systematic review and meta-analysis in England covering the period of 1950-2009 on the incidence of schizophrenia and other psychoses to determine the extent to which the rates varied, in particular focusing on gender, age and less accepted epidemiological gradients such as ethnicity and migration. The results echoed previous findings of elevated rates of disorders in ethnic minority groups, in particular African Caribbean and black African, when compared with the white British population. In schizophrenia the black Caribbean (pooled RR: 5.6; 95\% CI: 3.4-4.5; $n=5$ ), black African (RR: 4.7; 95\% CI: 3.3-6.8; $n=5$ ) and South Asian groups had a smaller risk ratio (pooled RR: 2.4; 95\% CI: $1.3-4.5 ; n=3$ ). Overall this study maintains that the incidence of schizophrenia has remained stable over 60 years. Of concern is the fact that this trend of elevated rates in African Caribbean group has continued (Pinto et al., 2008) despite efforts by Delivering Race Equality (Department of Health, 2005), a government initiative to reduce these figures.

Cognitive behaviour therapy (CBT) is now a plausible treatment of choice for schizophrenia and associated psychotic symptoms (NICE, 2009, 2014). A number of systematic reviews and meta-analyses (Velthorst, et al., 2015; Wykes et al., 2008; Zimmermann et al., 2005) have concluded that CBT is effective in treating people with schizophrenia and when not, it still appears to show benefits such as reducing client distress (Berry and Hayward, 2011). However, clients from some BME groups (e.g. African Caribbean and Black Africans) have shown higher drop-out rates and poor outcomes in comparison with their white counterparts (Rathod et al., 2005).

Cultural factors play an important part as beliefs, behaviours, emotions and even psychological responses to situations will vary depending on the cultural background of the client. Moreover, CBT has been criticized as being 'Eurocentric' (McCulloch et al., 2005) and therefore may not fit in with other cultures. In western cultures, predominant schema values individualism. In Eastern or collectivist cultures the predominant schema is being part of a group (Hays and Iwamasa, 2006; Tseng et al., 2005). Additionally, Laungani (2004) identified more core values that distinguish western and eastern cultures. Furthermore, perceptions of illness in schizophrenia differ across 
cultures as emphasized by McCabe and Priebe (2004) who argue that a biological explanatory model was related to enhanced treatment satisfaction but not to treatment adherence.

Despite significant findings regarding the efficacy of CBT on treatment resistant schizophrenia, negative and positive symptoms, Lynch et al.'s (2009) meta-analytical review of CBT for schizophrenia, bipolar and major depression, concluded that 'CBT is no better than non-specific control interventions in the treatment of schizophrenia and does not reduce relapse rates...'.

Lynch et al.'s (2009) critical viewpoint on well controlled trials should be taken with caution; the authors highlight the impact of blinding of assessors and controls in the studies they reviewed as one aspect they focused on, and posit that in studies where blinding was evident the outcomes of therapy were inconclusive. Furthermore, Lynch and colleagues criticize the meta-analysis procedure and the studies included in them, by re-analysing blinding as a moderator variable and conclude that current evidence for CBT is less convincing in treating schizophrenia. This review contradicts positive analyses already mentioned above. In view of the 2009 NICE guidelines of schizophrenia, the updated evidence base supported CBT for schizophrenia. Lynch et al.'s criticism of current CBT for the schizophrenia evidence base has already been challenged by the proponents of its efficacy, e.g. Kingdon (2010), who refutes the findings of this review and concludes by emphasizing that Lynch et al.'s exclusion of relevant studies may have indeed influenced negative outcomes.

The Department of Health has invested substantial funds for Improving Access to Psychological Therapies (IAPT). However, there appears to be poor access to such services for BME groups (Haddock et al., 2014). Furthermore, according to an executive summary published by NHS Digital (2017), as of October 2017, even those who have access to IAPT services, there appears to be a much more significant drop-out rate in BME clients (84.3\%) compared with White clients $(21.5 \%)$. Why are those of a BME background not completing psychotherapies as much? This may be linked to therapies that are currently delivered being grounded in Western views. Socio-demographic factors and cultural background influence perception of symptoms of mental illness and hence engagement with services. Cultural adaptations and understanding of ethnic, cultural and religious interpretations is an area that currently remains under-developed (Rathod et al., 2008).

A study has investigated the use of a culturally adapted CBT (CaCBTp) compared with treatment as usual (TAU) with BME populations diagnosed with schizophrenia. They showed that CaCBTp was much more effective (Rathod et al., 2013). But what is it that may make CaCBTp more effective? A more recent meta-analysis has similarly shown significant post-treatment effects of culturally adapted treatment (Rathod et al., 2017). The theme of therapeutic alliance was investigated within this meta-analysis and was shown to have a positive effect on outcome measures of symptom severity. The current paper aims to identify how client-initiated self-disclosure may play a role here in a therapeutic alliance.

\section{Self-disclosure}

Self-disclosure is a purposeful expression or a bridge in understanding a client's state at a particular moment. It is a way of engaging clients in treatment and is reciprocal in a therapeutic rapport. According to Simone et al. (1998) self-disclosure is defined as 'a conscious, intentional technique in which clinicians share information about their lives outside the counselling relationship'.

Traditionally, the attitude towards self-disclosure in psychotherapy has been based on the Freudian concept with great emphasis on psychoanalysts to be:

'impenetrable to the patient... reflect nothing but what is shown to him' (Freud, 1912). 
In the conventional therapist-client relationship, it is the client who discloses. Occasionally a therapist will decide whether to disclose something personal or not. Luft and Ingham's (1955) Johari window ${ }^{1}$ may be useful in shedding light on the aspects of the self that therapists are prepared to disclose, i.e. those aspects that are known to self and others. In contrast, any aspects that are not known to others (our emphasis: in this case the client) the authors postulate that the individual (our emphasis: a therapist) maintains a façade.

A more recent systematic review has shown that $90 \%$ of practitioners report that they self-disclose information about themselves to clients (Henretty and Levitt, 2010). From the use of participants' ratings of vignettes, it has been shown that ratings of self-disclosing therapists were more positive including ratings of attractiveness and empathy (Kaufman, 2016).

There are some therapy approaches that actively advocate the use of therapist self-disclosure (TSD) including social constructionist family therapy (e.g. Freedman and Combs, 1996). In such therapy sessions, the therapist is encouraged to put comments into a personal context. In contrast, however, other forms of therapy such as CBT have had little research focus on TSD. Goldfried and colleagues (2003) are one of very few who have, as of yet, explored the benefits of TSD in CBT. They have concluded that it is both a useful and appropriate therapeutic intervention within CBT (Goldfried et al., 2003).

\section{Self-disclosure and BME populations}

The literature suggests that those from a BME background may be more reluctant to disclose in a therapeutic environment. Anderson et al. (2009) has shown that African Caribbean people in the UK can be secretive and reluctant to disclose and tend to be cautious when talking to outsiders about their personal problems. This can be an issue for therapy and may hinder the outcomes if the patient is reluctant to disclose information that may be useful to the session.

BME clients also appear to fear being misinterpreted and misunderstood when disclosing information and in some cases fear incarceration as a result (Fernando, 1988). Furthermore, African Caribbean patients have elevated issues of mistrust and dissatisfaction with main stream statutory services (Keating, 2007; Keating et al., 2002; Keating and Robertson, 2004; Raleigh, 2000).

One way to possibly help BME patient disclosure is if the therapist also discloses. It is of paramount importance to distinguish between therapist self-disclosure as defined by Patterson and Hidore (1997). The authors postulate that disclosure is facilitated by the therapist and assume that most clients will be willing to talk about themselves and their problems when they come to therapy. Although this may be the case for the predominantly white population, working across cultures indicates a more mixed consensus. In their review, Henretty and Levitt (2010) have shown that there are mixed views of self-disclosure by different ethnicities. For example, Mexican clients appeared to not show preference for TSD where as Americans did (Cherbosque, 1987). In this study Mexican clients perceived TSD as a characteristic of therapists with less expertise. In a more recent study, however, Mexican-American men who were having mandated therapy described the importance of TSD in making therapy a more comfortable place to disclose

\footnotetext{
${ }^{1}$ The Johari window was developed by two psychologists, Joseph Luft and Harrington Ingram, in 1955. This model is used as a disclosure and feedback model of self-awareness and an information processing tool. There are four Johari window perspectives in terms of whether information is known or unknown by the individual and whether the information is known or unknown by others. This refers to self and others. The first window gives perspective of what is known by the individual about him/herself and is also known by others (this could be, e.g., information individual publicizes like a CV). The second window gives the perspective of what is unknown by the person about him/herself but which others know (this is termed a blind spot). The third window perspective refers to what the individual knows about him/herself that others do not know (this could be skeletons in the closet) and the fourth window perspective refers to what is unknown by the individual about him/ herself and is also unknown by others. The Johari window can be used to increase the open area (what is known by individual and others through the process of disclosure which reduces the hidden area. Other strategies such as observation of others can reduce unknown areas including self-discovery. Shared discovery can be used to reduce unknown area.
} 
(Bitar et al., 2014). Mixed results with this particular BME population have been reviewed by Sue and Sue who suggest that at first, Mexican-American individuals may perceive therapists utilizing TSD as having less expertise but as familiarity with the therapist increases over time TSD may be deemed more appropriate by the client (Sue and Sue, 2003).

African American clients appear to indicate a higher preference for TSD than Caucasian clients, specifically relating to TSD of topics including personal feelings, sexual and professional issues, and of success or failure (Cashwell et al., 2003).

With regard to South-Asian views on TSD, it has been shown that it can be perceived to have both beneficial and hindering effects, although it seems to have an overall positive impact for South-Asian patients (Patel, 2016).

There therefore appears to be a mixed consensus of TSD with BME clients. This is a theme that was evident in our 2010 study. The current paper thus aims to investigate this theme in more depth. It aims to identify what, in particular, are the views of a range of both BME clients and lay people and also therapists on TSD, and how this may affect psychotherapies in the future.

\section{Method}

\section{Study design}

The study adopted an over-arching qualitative methodology informed by an ethnographic approach (Richie and Lewis, 2003). In this case, qualitative methodologies were particularly suited to 'exploring and understanding the meaning individuals or groups ascribed to a social or human problem' (Creswell, 2009). Furthermore, the qualitative design was felt most appropriate given that empirical studies have demonstrated the efficacy of CBT in psychosis within the white population and there is paucity on ethnic minority patients' experiences of this intervention (Forchuk and Roberts, 1993). Leininger's (1985) definition of qualitative research describes several methods and techniques used to investigate the phenomenon at hand. These include observational, documentation, analysis and interpretation of attributes, patterns and underlying meanings.

Given the study's aim to elicit any cultural factors that influence the patient's response to their illness and therapy, this approach to data gathering allowed focus on understanding the perspective of people participating in the study within their cultural context (Spradley, 1980). Acknowledging the sensitive nature of the topics to be explored and the vulnerability of the service users, in depth face-to-face semi-structured individual or group interviews with participants were performed. In addition, focus groups (as a form of group interview) were used to clarify, explore or confirm ideas with a range of participants on a 'predefined set of issues'. The principle of 'emergent design' was followed when respondents raised issues that required further exploration or verification (Creswell, 2009; Polit and Hungler, 1999), These points were then tested appropriately with subsequent participants. Occasional telephone or email communications with participants enabled clarification of areas of uncertainty when the data were analysed (Rathod et al., 2010).

\section{Study centres}

The study was conducted in two centres in the UK: Hampshire and London. Hampshire represents a mixed inner city area and a small rural population of ethnic minorities. Therefore the issues of urban and rural areas were addressed (Rathod et al., 2010). Within Hampshire three sites agreed to participate, representing three different areas. These were Southampton, Portsmouth and Winchester. Study participants and rationale for their selection is included in the following 'Study participants and rationale for their selection' section. Moreover, given that this was a purposive target sampling we were aware that research in ethnic minorities has had difficulties recruiting in the past for several reasons, namely, BME populations tend to be concentrated in urban cities such as London, the Midlands and northern England. They are widely dispersed, 
making it difficult to consider cluster sampling. It was considered appropriate to use nonprobability sampling rather than probability sampling as it allows for in-depth examination of information from the subjects (Phiri, 2012).

\section{Study participants and rationale for their selection}

BME groups are classified (Office for National Statistics, 2001) into the following categories: Asian and South Asian (Indian, Pakistani, Bangladeshi) and Chinese and the Black and Black British (African, Caribbean). The Chinese group was excluded because its numbers were insignificant in the original insight study. The Indian group was excluded after discussion within the initial study group as this might have over-extended the study. The contrasts that would be drawn out between African Caribbean and South Asian Muslim groups and with the white population led to their selection.

The research team actively recruited those who were defined as Black Caribbean, Black British, Black African and Pakistani or Bangladeshi. Ethnic membership was verified with the participants.

Group 1. Comprised African Caribbean lay people and those patients with psychosis with a need for, but without experience of CBT and those with experience of CBT. The term African Caribbean in this study is used to describe lay people or service users with African ancestry who migrated from the Caribbean isles, African group, second generation Black British, and some from mixed race parentage. The rationale for selecting this ethnic group follows previous and current studies on ethnicity that concluded that there were disparities in health provision (Specialist Library for Ethnic and Health, 2009), significantly higher rates of schizophrenia in the UK (Sharpley et al., 2001) when compared with the general population (Pinto et al., 2008) and those in English speaking Caribbean. Moreover, this group is over-represented in mental health settings and especially in inpatient services, with high rates accessing services through detention on the Mental Health Act sections (Lombard, 2008).

Group 2. Comprised South Asian Muslim participants including Pakistani and Bangladeshi lay people and those patients with a need for, but without experience of CBT and those with experience of CBT - to reflect the diversity between different groups. The choice of South Asian Muslim groups was based on inclusion of ethnic groups of similar socio-economic status but with differing needs when compared with the Caribbean group in terms of language and religious background. Unlike the African Caribbean group, the South Asian Muslim group is under-represented in mental health services raising debates about reasons for not using these services (Patel and Andrade, 2003). Although the Pakistani and Bangladeshi Muslims share Islamic religion as a common factor, there are differences with respect to their culture, language, social class or caste and philosophical beliefs (Rack, 1992). By exploring within four groups Black British, Black African/African Caribbean, Pakistani, and Bangladeshi - challenges can be identified that can inform generic adaptation for culturally diverse groups (Department of Health, 2003; Nazroo, 1997) whilst retaining the principle that the individual's cultural needs must still be taken into consideration.

Group 3. Comprised cognitive behaviour therapists with experience of working with the defined BME groups. Although this was a mixed group, the majority of therapists were predominantly white.

Group 4. Comprised mental health practitioners (MHP) with generic experience of working with the defined BME groups. This group included a mixed ethnic profile reflecting the current workforce in the healthcare services.

Rathod et al. (2010) Reproduced with permission, Cambridge University Press.

\section{Sampling and recruitment}

Purposive, targeted sampling was adopted to recruit the study participants who consisted of service users, lay members of the respective ethnic communities, mental health practitioners and therapists. Sample size was determined pragmatically by three considerations, namely: 
(1) The likely number of informants required to gather meaningful data, typically at least 6-8 per focus group (see Krueger, 1994; Merton et al., 1990; Stewart and Shamdasani, 1990).

(2) The extent to which data were saturated and no 'new information' was being generated.

(3) The availability of participants from the ethnic groups concerned. Participants received information leaflets outlining the study; the leaflets were also translated into Urdu language for the Pakistani participants.

\section{Recruitment of participants}

Recruitment of individuals with schizophrenia/schizo-affective disorder or delusional disorder was through the local mental health teams, inpatient services and lay participants were recruited from local BME communities. Patients were approached only after their consultant psychiatrist had given permission. Written informed consent was obtained from participants before the interviews were conducted (Rathod et al., 2010). The inclusion and exclusion criteria can be found in Appendix 1.

\section{Recruitment of lay members, therapists and mental health practitioners}

Community Development Workers (CDWs) and local BME community organizations were approached to identify potential lay participants. Presentations and discussions with relevant organizations were arranged by the researcher as part of the recruitment process. The recruitment process for CBT therapists and MHPs involved approaching mental health services and local community mental health teams in Hampshire and London centres (Rathod et al., 2010).

This study was adopted by the North London and West Hubs of the Mental Health Research Network. Their Clinical Studies Officers (CSOs) assisted with recruitment.

Focus groups and individual interviews were conducted at a time and place convenient to the participants. Various definitions of focus groups exist, including that of Kitzinger (1995), who describes it as:

'... a form of group interview that capitalises on communication between research participants in order to generate data.'

Although group interviews are often used simply as a quick and convenient way to collect data from several people simultaneously, focus groups explicitly use group interaction as part of the method.

Notwithstanding their possible criticism of subjectivity and not being a replicable technique, they are the best method in this study. When compared with the mechanical nature of survey-based methods, interviews provide for emotional quality based on the personal qualities of the researcher (Kvale, 1996). Furthermore, focus groups offer some level of support, allowing people to open up. By exploring the attitudes and needs of participants, the researcher can learn a lot by watching the dynamics of the group. Conversely, focus groups do not discriminate against illiterate people and can operate within a given cultural context (Rathod et al., 2010).

A total of 38 interviews or focus groups consisting of individual semi-structured interviews with patients with a diagnosis of schizophrenia, schizo-affective, delusional disorders or psychosis $(n=15)$; focus groups with lay members $(n=52)$; CBT therapists $(\mathrm{n}=22)$ and mental health practitioners $(n=25)$ were conducted by the researcher (P.P., a therapist from a BME background) between May and December 2008. All groups and interviews were audio-recorded, and transcribed. Transcripts were coded and anonymized. Access to data was limited to the researcher and the research team. Field notes were taken by the researcher noting non-verbal communication and behaviours. Focus group discussion data were analysed according to the 
Box 1. Summary of main themes explored through interviews and focus groups

\section{Guide themes with health professionals}

- Experiences of conducting CBT with BME communities

- Good and negative experiences, useful tips

- Whether and how the CBT manual is used during therapy with BME patients

- Similarities and differences in BME communities

\section{Guide themes with service users who experienced CBT}

- Understanding of causes and experience of psychosis and its treatment

- Comparisons between family, friends, psychiatrist and therapist views of this mental health problem

- Actions taken by patient/family/friends prior to referral for CBT

- Experiences of referral, accessing services and therapy associated with CBT - other strategies used by patient

- Whether therapist/doctor understood their problems

- What therapies were helpful?

- Whether patient would recommend CBT to another person, - reason for decision

\section{Guide themes with service users with no experience of CBT}

- Understanding of causes and experience of psychosis and its treatment

- Comparisons between family, friends and therapist views of this mental health problem

- Actions taken by patient/family/friends to seek help or manage the problem (e.g. when to go to doctor or spiritual healer, etc.)

- Whether therapist/doctor understood their problems

- Views on CBT (if known)

- What therapies are helpful?

\section{Guide themes with lay BME community members}

- Understanding the cause, origin and manifestation of 'psychosis'

- Description of psychosis (typical expressions or words used)

- How the community judges is the best way to treat psychosis

- How they support the person with psychosis

- How they react to the person (male or female) with psychosis

- What their views are on the interventions of doctors and mental health practitioners

From Rathod et al. (2010), reproduced with permission from Cambridge University Press.

principles recommended by Krueger (1994). The data were largely descriptive with most themes emerging in response to the interview guides. The interview guide themes are outlined in Box 1. The data were analysed by systematic content and question analysis (Morse and Field, 1996), unlike other types of thematic content analysis that place emphasis on frequency of words and utterances (Hsieh and Shannon, 2005). Analysis involved the researcher immersing himself in data by reading the interview transcripts carefully (sometimes re-read several times) and identifying emerging themes and categories (Simons et al., 2008). NVivo 8 (computer-assisted qualitative data analysis software) was used to manage and explore the qualitative data in depth (Rathod et al., 2010).

Content analysis is a systematic and objective method for identifying, analysing and describing the data in depth. It involves coding participants' open-ended verbatim into categories that summarize data and is sometimes treated as similar to thematic approaches (Braun and Clarke, 2006). The benefits of using a computer package (NVivo) included accuracy, speed and consistency. Moreover, Lewins and Silver (2007) postulate that packages do provide an audit 
trail for analysis and facilitate the linking of ideas to data. This cognitive map provided a pathway of how we got to the broader emergent themes. NVivo was the most appropriate package following Lewins and Silver's (2007) step-by-step guide to using software in qualitative research. The researcher attended training on qualitative software; this was useful in making a choice of suitable package. The NVivo system was useful in organizing emerging themes using free nodes that were later categorized. According to Braun and Clarke (2006), themes capture essential aspects of data in relation to the phenomenon under investigation. The flexibility of thematic analysis allows the researcher to determine the emergent themes and prevalence; this is an advantage of content analysis. Furthermore, content analysis can generate anticipated insights and its findings can be comparable to similar studies undertaken with a similar framework. Notwithstanding its strengths, there are limitations with content analysis; in particular, sections of excerpts that are both representative of data and compelling to the reader can be challenging. It has limited interpretive power beyond mere description if not used within an existing theoretical framework.

Thematic content analysis was utilized to identify themes, concepts and meaning, and according to Braun and Clarke (2006) it should be seen as a method in its own right and not as a process of qualitative research. A fundamental strength of content analysis is the ability to enable the qualitative researcher to investigate sensitive topics without altering the phenomena under investigation. Furthermore, its data can be subject to re-analysis therefore allowing for reliability checks. Unlike grounded theory and interpretive phenomenological analysis (IPA), thematic analysis is a relatively easy method to use and is accessible to a wide audience. It can also be useful for informing policy development (Braun and Clarke, 2006).

Thematic analysis differs from other analytic methods in qualitative data and the following were considered: discourse analysis, IPA (Smith et al., 2009) and grounded theory. Grounded theory (Corbin, 2017) and IPA were not suitable for this study because they are both theoretically bounded (Braun and Clarke, 2006). Moreover, IPA is primarily about understanding people's everyday (subjective) experiences of reality in great detail so as to understand the phenomenon under investigation. Whereas the grounded theory analysis approach primarily aims towards theory generation and development (Braun and Clarke, 2006), a practical difficulty relates to deciding when categories are 'saturated' or indeed when theory is sufficiently developed. The focus of our study was not to develop a model of social processes as suited by grounded theory. Discourse analysis focuses on use of language, in particular how certain issues are constructed in people's accounts, and considers the function of such dialogue or discourses in the context of ongoing interaction (Willig, 2008). Although an ideal approach, it best suits small sample sizes because of its demanding analysis. To conclude, thematic analysis involves searching through a data set to identify repeated themes of meaning and was an appropriate choice for our research question.

Triangulation of themes and concepts was undertaken to compare and contrast the data from the different participating groups. This enhanced the reliability and validity of the analysis. A sample of transcripts was independently reviewed by three other researchers to check the reliability of the interpretation, coding and inter-relationship of themes. Following review of sample transcripts, three independent researchers met and compared emergent themes.

The presence of African Caribbean members on the team enabled these communications to be 'translated' and explained. The researcher met on a regular basis with the research team (S.R., D.K., M.G.) and also with the Steering Group who had varied expertise and experience. Expertise on qualitative methodology was provided by M.G. (Rathod et al., 2010).

\section{Finding related to TSD theme}

The overall findings can be found in the Rathod et al. (2010) paper. The primary objectives were 'to gain meaningful understanding concerning the way members (lay and service users) of the above minority communities typically view psychosis, its origin, and management'; (ii) to elicit 
Table 1. Rathod et al. (2010), reproduced with permission from Cambridge University Press

\begin{tabular}{lccc}
\hline \multicolumn{2}{l}{ Attributions to psychosis } & & \\
\hline Theme: Causation & Afro-Caribbean & Bangladeshi & Pakistani \\
\hline Previous wrong doing & +++++ & +++++ & +++++ \\
Supernatural beliefs & +++++ & +++++ & +++++ \\
Social factors & ++++ & ++++ & ++++ \\
Biological & +++ & +++ & +++ \\
Being arrested & +++ & & ++ \\
Drug induced & +++ & & \\
\hline
\end{tabular}

those cultural influences, values and attitudes that shape a client's degree of participation and response to CBT; (iii) to elicit from $\mathrm{CBT}$ therapists and mental health practitioners from these communities their experiences and interpretations of the way a client's culture influences their attitude and response to CBT; (iv) to identify those strategies that CBT therapists and other MHPs identify as being supportive or non-supportive with BME clients; and (v) to produce a practice guide and recommendations for training. Emergent themes relating to the participant's explanatory models of what causes mental illness, in particular psychosis, are summarized in Table 1. As the current paper focuses on one specific theme (client-initiated therapist self-disclosure) the thematic analysis of this is evident below.

\section{Rationale for self-disclosure: the why?}

TSD seemed to be of great importance to the majority of the sample. The reason for this was twofold; first, it signified that the therapist or clinician was treating the client as a human being and 'not just another number'. This means that the client is judging and using this process as a vehicle to establishing a rapport with the therapist.

Secondly, by not being hesitant and answering the question at hand the client assumed they could trust this therapist and therefore took the therapeutic rapport to another level. The client will be seeking a degree of spontaneity and determines the manner and speed with which response is given. When therapists are assessing their clients they need to be aware that the client is also assessing them. Clients tend to come to a conclusion earlier than the therapist. Here they are eliciting the degree of trust. However, when a therapist was defensive in their response and/or declined to answer the posed question on self-disclosure, the BME client revealed minimal information or was less responsive in therapy.

This theme of self-disclosure although consistent in the African Caribbean and South Asian Muslim groups in our study was explicit in the Afro-Caribbean group owing to their perceptions and assumptions of being portrayed as 'them and us', 'I am seen as just a number'. The following narrative typifies this:

\section{'If I'm going to tell you about myself, I want to know something about you.'}

This was confirmed by some service user participants in this study who admitted to testing professionals by asking them personal questions in order to ascertain whether they could trust them. Ethnic minority groups are wary of clinicians, fearing they might be misunderstood and misinterpreted. There may be a link between this client-initiated therapist self-disclosure and mistrust for statutory services and dissatisfaction (Bhugra, 1997; Bhui, 1997).

\section{'Testing' of therapists}

Interestingly this 'testing' was done to all clinicians regardless of their race and ethnicity. First impressions count and can be a litmus test as to whether the client engages with the therapist 
Table 2. Cultural barriers to therapy

\begin{tabular}{l}
\hline Cultural barriers to therapy \\
\hline Mistrust of services/practitioners \\
Worries about confidentiality/breach \\
Poor information on psychological therapies/accessibility \\
Language and terminology leading to being misunderstood \\
Fear of being stigmatized \\
Previous experience \\
Stereotyping by therapists \\
Doubt regarding CBT being empowering enough \\
Lack of understanding of cultural norms, values by \\
therapist \\
(Cultural incompetence) or Euro-centric approach \\
Clinician's beliefs in the power of drugs \\
Faith/spirituality and religion \\
Individualism vs collectivism \\
Gender issues \\
Racism/colonial history \\
Interpretation problems \\
Financial implication \\
Practical issues, e.g. environment of therapy, transport
\end{tabular}

or clinician. Furthermore, the level of engagement maybe dependent on whether the therapist passes that test.

Pertinent to the African Caribbean participants was the issue of trust and its association with assessing the therapist if they were trustworthy, genuine and would relate to them as a person. The study showed that African Caribbean communities were sensitive to issues relating to trust. Cultural barriers to therapy including mistrust are summarized in Table 2. Mistrust stems from years of dissatisfaction with the mainstream services and their previous experiences and encounters with services (Keating et al., 2002; Keating and Robertson, 2004; Keating, 2007; Parkman et al., 1997; Raleigh, 2000).

African Caribbean service user participants admitted to asking therapists tricky questions just to see how they would respond to them. For instance, they would ask questions such as, 'Do you have children?' or 'Where do you live?'. Some questions were so subtle such that the therapist was unaware they were being tested. Example 1 illustrates a situation whereby a therapist 'reflects nothing but a mirror'. In contrast, Example 2 illustrates some elements of therapist self-disclosure.

The fear of feeling misunderstood and misinterpreted was identified as an influencing moderator that contributed to this ethnic group feeling vulnerable and therefore testing practitioners at onset. It is important to note that therapists are not singled out when it comes to seeking self-disclosure, the African Caribbean participants reported that they would do this to their friends as well.

Similar tactics of 'testing' therapists existed within the South Asian Muslim participants. Of particular importance was the subject of social class and cast of the therapist if they belonged to the Asian ethnic group; the following narrative illustrates this:

'... so they asked me whether I was from India or from Sri Lanka ... because they are saying confidential things... it's more of a security issue...'

'...for Asians it's very tricky because the caste system is there and I see it from the nurses that they dread the question of which caste are you from ... it can be very difficult if you are from a lower caste...' [F4, CBT therapist focus group interview]

This was also reported by therapists who have worked with refugees, asylum seekers and interpreters. They would also want to know the therapist's religious beliefs. In some cases the age, marital status and the number of children the therapist had was important in particular 
to some first generation lay participants. They related that talking to someone who had for instance, the same number or more children than them was perceived as experienced, credible and an expert. Therefore, they assumed that such a practitioner would be able to relate to their needs more than a practitioner who was not a parent. The following narrative details this:

'... one psychologist recently told me that she was confronted by a woman ... "do you have children?" ... and clearly it was coming from one-upmanship. "I have three children", the psychologist happened to have four, so she had credibility, is what she said, "yes I have four children." If she had said, no and hadn't disclosed, she was gone, there wouldn't have been a relationship ... clearly this was a big status symbol and very important ...' [F4, CBT therapist focus group interview]

\section{Therapists' experience of self-disclosure}

A further important factor related to this theme was the therapist's experience and comfort in self-disclosing. Some African Caribbean service user participants reported that they intentionally used subtle questions on clinicians and/or therapists. We therefore asked cognitive behaviour therapists in focus group interviews how they dealt with self-disclosure as initiated by clients. A few therapist participants in response, reported that they felt secure in themselves and did not find disclosing a personal issue a problem as long as this was appropriate and within therapeutic boundaries. In contrast, those therapists who had responded in a defensive manner to questions of self-disclosure in particular with the African Caribbean patients reportedly found it difficult to engage their clients (Rathod et al., 2010).

Where therapists felt secure about themselves they did not have problems about self-disclosure and some reported that this did promote engagement in treatment. One narrative outlines this:

'I don't have issues with disclosure provided you don't breach any basic safety, so I will not disclose where I live... what bus I take. But that's just personal common sense. That's just our code of practice as well. I think disclosure can have a very therapeutic use and I certainly use it very often' [F2, CBT therapist focus group interview]

In response to the quote 'If I'm going to tell you about myself, l want to know something about you', the respondent further states:

'And I think yes they are right, very often we do ask lots of questions and when people ask you, "where are you from?" ... and some people say, "why do you want to know that? ".....well you know, I don't have an issue with telling people that I am Spanish. ... I think I do certainly use a lot of self-disclosure, I guess therapeutically within guidelines.' [F4, CBT therapist focus group interview]

Thus it appears that there were two main elements relating to the theme of client-initiated TSD; that is, the way in which therapists responded to the 'tests' given by BME clients was as important as the content of their response. Furthermore, the more comfortable and willing a therapist was in disclosing the better engagement appeared to occur in treatment.

\section{Discussion}

Overall, the results of this thematic analysis appear to show that there is a general consensus among BME client groups for TSD. More specifically, they appear to encourage this by initiating the disclosure themselves through 'testing' their therapist. However, these clients do not find the content of the disclosure as important as the way in which the therapists react and respond. 


\section{Should a therapist self-disclose?}

From the thematic analysis conducted here, it appears that the general consensus of the BME sample investigated is that TSD is important. Participants have shown that it aids trust and the overall relationship of client and therapist. Goldsmith et al. (2015) have shown that therapeutic alliance has a strong causal link with an increase in number of sessions taken by the client and a decrease in symptoms. Therefore, it suggests that CBT therapists engaging with BME clients with schizophrenia or psychosis should embrace client-initiated TSD and thus strengthening the therapeutic alliance rather than avoiding it.

\section{What to disclose}

The findings of this thematic analysis appear to show that BME client groups advocate and even initiate TSD. Therapists' responses to client-initiated self-disclosure should be sensitive and take into account their cultural background. It is not the content but the context with which the response focuses on. So if a therapist tactfully responded to the client without divulging anything personally but was sensitive in their response, the client felt reassured. The other reason raised by the participants questioned the way clinicians expect to know much about the clients, when they themselves do not want to self-disclose. It is imperative to develop therapeutic rapport based on reciprocity when dealing with self-disclosure issues.

\section{Risks of therapist self-disclosure}

'Does my experience match that of the patient or am I being indulgent?'

Levitt and colleagues have argued that disclosures that convey similarity between the therapist and client are associated with fewer post-session clinical symptoms and also fewer interpersonal problems (Levitt et al., 2016). There are, however, situations where a therapist's experience although similar to a client's experience may not match in many respects; for instance, a client may state that his or her mother has died. The therapist may empathize by disclosing that they do understand because they too lost a parent a while ago, and can relate to what the client may be experiencing. To play the devils' advocate here, what happens to that form of therapist self-disclosure when supposedly a client then replies, 'I'm glad the old nag is gone, I hated her'?

Therapist disclosure of similar experiences with their client as explored by Patterson (1985) purports the disadvantages may lead the client to query the expertise or competence of the therapist. In particular the perception that problems may not be taken seriously or are being undermined, for instance 'look at me I got through that problem, you too should be able to get over this soon', could result in the client perceiving the therapist as having unrealistic expectations of them. Consequently, the client may feel unmotivated.

Furthermore, there is a danger that the therapist may over-identify with a client's problems and thus lose focus on the actual presenting problem by focusing on a less pertinent issue. Conversely, clients may be motivated that they have something in common and assume that the therapist will be able to relate to their problem. Similarly, Gondim (2006) provides a counselling viewpoint on self-disclosure and argues that when used appropriately this technique can be helpful in the therapeutic process. This is echoed by Beck (2007): she relates using self-disclosure with specific clients she believes would benefit from it. One example she notes in her blog relates to a client with perfectionist standards and discloses the standards she applies to herself and her children. The unknown area in the Johari window in Beck's scenario appears to be when she talks about her son with severe learning disabilities to a client with inferiority issues. She then explores with the clients how her self-disclosure might apply to them. Here is an extract from her blog 
'I don't use self-disclosure with every patient but I do with most ... often gives them a different way of thinking about their problems ...goes a long way in strengthening our relationship when patients recognize ... I am a human being ... willing to share something of herself to help them. ' (Beck, 2007)

For some it may harness hope that they will get better, while for others this form of therapist disclosure may normalize their distress and be less stigmatizing. These variables will require investigating in future studies to test their effectiveness.

Therapists should err on the side of caution when matching their experiences to the client's and consider whether this is relevant and would act as a bridge rather than a barrier to engagement in the therapeutic process. Consequently, their disclosure might not be mistaken for indulgence.

'If I'm going to tell you about myself, I want to know something about you.' [interview with African Caribbean participant]

It has been previously mentioned that there is an issue of blurring the boundaries between client and therapist. The above excerpt further indicates that issues of self-disclosure can be problematic. Most practitioners are familiar with the concept of self-disclosure. The most common form involves voluntary disclosure of one's personal situation or illness to another, in this case a patient or client. Disclosure of feeling and insight has been shown to be rated as high in quality by clients as opposed to alternative disclosures of fact (Pinto-Coelho et al., 2016). It is assumed that the role of disclosure will be to instil hope (Fisher, 1994) to clients and hopefully nurture a process of openness and self-disclosure on their part, concurrently reducing any form of stigma and shame resulting from suffering with mental illness (Hyman, 2008).

There is always risk in self-disclosure and client-initiated TSD as highlighted earlier where the therapist's experiences does not match the clients'. When working with individuals who have difficult personality traits, or present with psychosis, therapists need to be aware that in some cases their personal experiences may be used against them and therefore should balance disclosure with genuineness. Interestingly, Rowan (2008) provides the following commentary on therapist self-disclosure when working with paranoid or delusional patients:

'Self-disclosure is almost obligatory with patients with serious difficulties in decentering, for example those suffering from delusions or paranoid ideation... If a therapist does not disclose what he or she thinks, patients will, in case of doubt, imagine, as the default option, that the therapist wants to trick them. Self-disclosure here is, therefore, almost the only action able to preserve the alliance.'

Therapists should weigh the cost and benefits of self-disclosure in such instances. Use of clinical supervision to support therapist to address TSD is recommended.

\section{Practical implications}

The thematic analysis conducted here has positive implications as it has shown that there may be a need for therapists to prepare and be comfortable in disclosing in CBT sessions, particularly when the client is of a BME background. As this appears paramount it may now require guidelines to aid therapists in how to successfully do this. This has been suggested continuously throughout the literature (e.g. Bitar et al., 2014; Henretty and Levitt 2010). As this is not yet a part of the training programme for therapists it may be essential for new trainees to ensure that the next generation of CBT therapists are able to aid the outcome of therapy for BME clients as much as possible by providing TSD. This may then reduce the current drop-out rates of BME clients with treatment. 
Example 1: 'reflect nothing but a mirror'

T: It's good to see you again; Safina before we start on this week's activities could tell me how your week has been?

S: Nothing much, same old, watching TV, the voices have been bothering me a lot. Other than that, nothing much.

T: Did you get any visits from the family or go out at all?

S: Yes, just my mother, she visited a couple times.

T: How did the visit go?

S: It was good to have company. What about you, how was your week?

$\mathrm{T}$ : My week was good, a bit busy?

S: What did you do?

T: Well, eh just a few bits here and there, but I'm wondering whether we can try and focus on you rather than me at the moment? Is that ok?

S: What did you do over the weekend usually?

$\mathrm{T}$ : Oh just the usual, you know, just keep self-busy and resting. Is it ok, to bring focus back to your overview?

S: Yes, ok, sure.

Example 2: some elements of disclosure

T: Hi Safina, it's good to see you again, let's begin by you telling me how you have been since I last saw you?

S: Same old, stayed in and watched TV.

$\mathrm{T}$ : Any interesting programmes you watched?

S: No, not really watched whatever was on, the weather has not been good.

T: It's that time of the year. Did you have any visitors over?

S: Yeah, my mum came to see me that was good. What did you do?

$\mathrm{T}$ : Well a bit of gardening

S: In this weather? Where do you live?

T: I live locally.

S: Where in Southampton?

$\mathrm{T}$ : Well, in the Eastleigh area.

S: Oh, that is where my kids go to school.

T: I see.

S: Do you have kids?

T: Oh yes I do.

S: How many kids do you have?

T: I have three kids.

S: I see, how old are they?

$\mathrm{T}$ : $\quad$ They range between 7 and 15 .

S: I have two kids; I have a daughter aged 10 years, and a 4-year-old son.

$\mathrm{T}$ : I notice your face smiling as you are talking about your children...

\section{Implications}

However, this study does have limitations. It was only conducted on a cohort of BME clients with specific mental health disorders (schizophrenia and psychosis). It may need to be conducted on other BME groups who require $\mathrm{CBT}$ such as those with depression to see if they share similar views on TSD and if they too initiate this. 
As this was a qualitative study, there is also a need for a quantitative study to investigate this further. If clients state that they prefer TSD and that it aids the outcome of therapy, such a study needs to show in a quantitative measure that client-initiated TSD is effective. A further study of CBT including or excluding client-initiated TSD from treatment may be useful to see if outcomes are greater for this population. If so, then it continues to aid the argument that it is a useful technique that should be used in CBT.

\section{Summary}

In conclusion, common sense prevails when basic values are maintained such as treating your patient as a human being. Sometimes getting into 'the others' shoes (metaphorically speaking) and treating them as you would like them to treat you if you were in a similar situation', is fundamental in developing a trusting relationship. How the therapist responds may be as important as the content of the response. For these clients, small elements of self-disclosure and warmth build trust and the feeling of being respected as equals. After all, the therapist-client relationship in cognitive behaviour therapy should be the meeting of two experts, the expert client and the expert practitioner who work collaboratively with the aim of helping the client to understand the development of their problems and develop alternative strategies of reducing distress. Given that this theme on client-initiated self-disclosure discussed here was limited to the Rathod et al. (2010) qualitative study, it is hoped that this article will stimulate a debate on this subject area and encourage researchers to investigate client-initiated therapist self-disclosure and its impact on engagement and outcomes in therapy.

Author ORCID. (D) Peter Phiri, 0000-0001-9950-3254

Acknowledgements. We would like to thank all the participants who contributed to our qualitative study.

Conflicts of interest. The authors have no conflicts of interest to declare.

Financial support. The project was funded by the Delivering Race Equality Programme: Clinical Trailblazers Project.

Ethical statement. Ethics approval for the study was obtained from the Southampton Research and Ethics Committee (B) REC: 08/H0504/5. Research and Governance approval was obtained from Hampshire Partnership NHS Foundation Trust, West London Mental Health Trust and Portsmouth NHS Trust.

Author contributions. P. Phiri wrote the first draft of the manuscript and this formed part of his doctoral thesis. All authors (P.P., S.R., M.G., H.C. and D.K.) contributed to manuscript revisions and approved the final version.

Key practice points

(1) First impressions count - when asked to disclose personal details, be aware of your behaviours and attitude.

(2) Hesitation to respond and a defensive stance could have implications for your rapport.

(3) Be aware of culturally derived behaviours, beliefs and attitudes.

(4) Enlist supervision for support and guidance on self-disclosure.

(5) Be sensitive in your response when working with patients from diverse groups with elevated levels of mistrust.

(6) Elements of self-disclosure and warmth can build trust and also give the feeling of being respected as equals.

(7) Training and development for clinicians on effective ways of applying self-disclosure with diverse clients is recommended.

(8) Further investigation on the efficacy of disclosure and managing risk factors in varied settings is suggested.

Derlega, V.J., \& Berg, J.H. (1987). Self-Disclosure: Theory, Research and Therapy. New York and London, Plenum Press. Farber, B. (2006). Self-Disclosure in Psychotherapy. New York, USA: Guilford Press.

Stricker, G., \& Fisher, M. (1990). Self-Disclosure in the Therapeutic Relationship. New York, USA: Plenum. 
Zur, O. (2007). Self-disclosure. In Boundaries in Psychotherapy: Ethical and Clinical Explorations, chapter 7, pp. 147-165. Washington, DC, USA: APA Books.

\section{References}

Anderson, A., Elam, G., Solarin, I., Gerver, S., Fenton, K., \& Easterbrook, P. (2009). Coping with HIV: Caribbean people in the United Kingdom. Qualitative Health Research, 19, 1060-1075.

Beck, J. S. (2007). Self-Disclosure in Therapy: Monday, 8 January 2007 at 8:59 pm and is filed under Cognitive Therapy, Judith S. Beck. Blog: available at: = http://www.beckinstituteblog.org/?p=146 (accessed 6 November 2010).

Berry, C., \& Hayward, M. (2011). What can qualitative research tell us about service user perspectives of CBT for psychosis? A synthesis of current evidence. Behavioural and Cognitive Psychotherapy, 39, 487-494.

Bhugra, D. (1997). Setting up psychiatric services: cross-cultural issues in planning and delivery. International Journal of Social Psychiatry, 43, 16-28.

Bhui, K. (1997). London's ethnic minorities and the provision of mental health services, pp. 143-166. London's Mental Health, London: King's Fund Institute.

Bhui, K. (2002). Racism and Mental Health: Prejudice and Suffering. Jessica Kingsley Publishers.

Bitar, G. W., Kimball, T., Bermúdez, J. M., \& Drew, C. (2014). Therapist self-disclosure and culturally competent care with Mexican-American court mandated clients: a phenomenological study. Contemporary Family Therapy, 36, 417-425.

Braun, V., \& Clarke, V. (2006). Using thematic analysis in psychology. Qualitative Research in Psychology, 3, 77-101.

Cantor-Graae, E., \& Selten, P. (2005). Schizophrenia and migration: a meta-analysis and review. American Journal of Psychiatry, 162, 12-24.

Cashwell, C. S., Shcherbakova, J., \& Cashwell, T. H. (2003). Effect of client and counsellor ethnicity on preference for counsellor disclosure. Journal of Counseling \& Development, 81, 196-201.

Cherbosque, J. (1987). Differential effects of counsellor self-disclosure statements on perception of the counsellor and willingness to disclose: a cross-cultural study. Psychotherapy: Theory, Research, Practice, Training, 24, 434-437.

Corbin, J. (2017). 'Grounded Theory'. Journal of Positive Psychology, 12, 301-302.

Creswell, J. W. (2009). Research Design, Qualitative, Quantitative, and Mixed Method Approaches (3rd edn). Los Angeles, USA: Sage Publications.

Department of Health (2003). Delivering Race Equality: A Framework for Action. London, UK: Department of Health.

Department of Health (2005). Delivering Race Equality in Mental Health Care: an action plan for reform inside and outside services and the Government's response to the independent inquiry into the death of David Bennett. Report no. 4393. London, UK: Department of Health.

Fearon, P., \& Morgan, C. (2006). Environmental factors in schizophrenia: the role of migrant studies. Schizophrenia Bulletin, 32, 405-408.

Fearon, P., Kirkbride, J. B., Morgan, C., Lloyd, T., Hutchinson, G., Tarrant, J., Fung, W. L., Holloway, J., Mallett, R., Harrison, G., Leff, J., Jones, P. B., \& Murray, R. M. (2006). Incidence of schizophrenia and other psychoses in ethnic minority groups: results from the MRC AESOP Study. Psychological Medicine, 36, 1541-1550.

Fisher, D. (1994). A psychiatrist's gradual disclosure. New York State, Office of Mental Health, OMH News, 6, 16. Available at: www.omh.state.ny.us/omhweb/news/

Fernando, S. (1988). Race and Culture in Psychiatry. Billing and Sons Ltd.

Forchuk, C. and Roberts, J. (1993). How to critique qualitative research articles. Canadian Journal of Nursing Research, 25, 47-56.

Freedman, J., \& Combs, G. (1996). Narrative Therapy: The Social Construction of Preferred Realities. New York, NY, USA: W.W. Norton \& Co.

Freud, S. (1912). Recommendations to physicians practicing psycho-analysis. In J. Strachey \& A. Freud (eds), The Standard Edition of the Complete Psychological Works of Sigmund Freud, volume XII (1911-1913): The Case of Schreber, Papers on Technique and Other Works (pp. 109-120). London, UK: Hogarth Press, 1958.

Goldfried, M. R., Burckell, L. A., \& Eubanks-Carter, C. (2003). Therapist self-disclosure in cognitive-behavior therapy. Journal of Clinical Psychology, 59, 555-568.

Goldsmith, L. P., Lewis, S. W., Dunn, G., \& Bentall, R. P. (2015). Psychological treatments for early psychosis can be beneficial or harmful, depending on the therapeutic alliance: an instrumental variable analysis. Psychological Medicine, 45, 2365-2373. doi: 10.1017/S003329171500032X

Gondim, P. T. (2006). Self-Disclosure - Underpinnings and Applications. Available at: = http://ezinearticles.com/?SelfDisclosure---Underpinnings-and-Applications\&id=366731 (accessed 23 August 2009).

Haddock, G., Eisner, E., Boone, C., Davies, G., Coogan, C., \& Barrowclough, C. (2014). An investigation of the implementation of NICE-recommended CBT interventions for people with schizophrenia. Journal of Mental Health, 23, 162-165. doi: 10.3109/09638237.2013.869571

Hays, P. A., \& Iwamasa, G. Y. (2006). Culturally Responsive Cognitive Behaviour Therapy: Assessment, Practice and Supervision. Washington, DC, USA: American Psychological Association. 
Healthcare Commission (2008). 'Count Me In Census'. Results of the 2008 national census of inpatients in mental health and learning disability services in England and Wales. Published by 2008 Commission for Healthcare Audit and Inspection.

Henretty, J. R., \& Levitt, H. M. (2010). The role of therapist self-disclosure in psychotherapy: a qualitative review. Clinical Psychology Review, 30, 63-77.

Hsieh, H., \& Shannon, S. E. (2005). Three approaches to qualitative content analysis. Qualitative Health Research, 15, 1277-1288.

Hyman, I. (2008). Self-Disclosure and its Impact on Individuals who Receive Mental Health Services. HHS Publication No. (SMA)-08-4337. Rockville, MD, USA: Center for Mental Health Services, Substance Abuse and Mental Health Services Administration. Available at: http://www.samhsa.gov

Jackson, J. S., Neighbors, H. W., Torres, M., Martin, L. A., Williams, D. R., \& Baser, R. (2007). Disentangling mental health disparities: use of mental health services and subjective satisfaction with treatment among Black Caribbean immigrants: results from the National Survey of American Life. American Journal of Public Health, 97, 61-67.

Kaufman, S. E. (2016). The effects of therapist self-disclosure of a mental health condition on client perceptions of the therapist. PCOM Psychology Dissertations, 365. Doctoral Thesis, Philadelphia College of Osteopathic Medicine DigitalCommons@PCOM

Keating, F. \& Robertson, D. (2004). Fear, black people and mental illness: a vicious circle? Health and Social Care in the Community, 12, 439-447.

Keating, F., Robertson, D., Francis, F., \& McCulloch, A. (2002). Breaking the Circles of Fear: A Review of the Relationship between Mental Health Services and African and Caribbean Communities. Sainsbury Centre for Mental Health.

Keating, F. (2007). African and Caribbean men and mental health. Race Equality Foundation.

Kingdon, D. (2010). Oversimplification and exclusion of non-conforming studies can demonstrate absence of effect; a lynching party? Psychological Medicine, 40, 25-27.

King, M., Nazroo, J., Weich, S., McKenzie, K., Bhui, K., Karlsen, S., Stansfeld, S., Tyrer, P., Blanchard, M., Lloyd, K., McManus, S., Sproston, K., \& Erens, B. (2005). Psychotic symptoms in the general population of England: a comparison of ethnic groups (the EMPIRIC study). Social Psychiatry and Psychiatric Epidemiology, 40, 375-381.

Kirkbride, J. B., Fearon, P., Morgan, C., Dazzan, P., Morgan, K., Tarrant, J., Lloyd, T., Holloway, J., Hutchinson, G., Leff, J., Mallet, R. M., Harrison, G., Murray, R. M., \& Jones, P. B. (2006). On behalf of the AESOP Study Group. Heterogeneity in incidence rates of schizophrenia and other psychotic syndromes: findings from the 3-center AESOP study. Archives of General Psychiatry, 63, 250-258.

Kirkbride, J. B., Errazuriz, A., Croudace, T. J., Morgan, C., Jackson, D., Boydell, J., Murray, R. M., \& Jones, P. B. (2012). Incidence of schizophrenia and other psychoses in England, 1950-2009: a systematic review and meta-analyses. PloS ONE, 7, e31660. doi: 10.1371/journal.pone.0031660

Kitzinger, J. (1995). Qualitative research: introducing focus groups. British Medical Journal, 311, $299-302$.

Krueger, R. A. (1994). Focus Groups: a Practical Guide for Applied Research (2nd edn). Los Angeles: Sage Publications.

Kvale, S. (1996). Interviews: An Introduction to Qualitative Research Interviewing. Los Angeles: Sage Publications.

Laungani, P. (2004). Asian Perspectives in Counselling and Psychotherapy. New York, USA: Brunner-Routledge.

Leininger, M. (1994) 'Evaluation Criteria and Critique of Qualitative Research Studies' in J.M. Morse (ed.), Critical Issues in Qualitative Research Methods (pp. 95-115). Thousand Oaks, USA: Sage Publications.

Lewins, A., \& Silver, C. (2007). Using Software in Qualitative Research: A Step by Step Guide. Los Angeles: Sage Publications.

Levitt, H. M., Minami, T., Greenspan, S. B., Puckett, J. A., Henretty, J. R., Reich, C. M., \& Berman, J. S. (2016). How therapist self-disclosure relates to alliance and outcomes: a naturalistic study. Counselling Psychology Quarterly, 29, 7-28.

Lombard, D. (2008). Healthcare Commission survey reveals increase in proportion of BME patients. Available at: http://www. communitycare.co.uk/articles/2008/11/28/110123 (accessed February 2009).

Luft, J., \& Ingham, H. (1955). The Johari window, a graphic model of interpersonal awareness. Western training laboratory in group development. Los Angeles: University of California.

Lynch, D., Laws, K. R., McKenna, P. J. (2009). Cognitive behavioural therapy for major psychiatric disorder: does it really work? A meta-analytical review of well-controlled trials. Psychological Medicine, 40, 9-24. doi: 10.1017/ S003329170900590X

Merton, R. K., Fiske, M. \& Kendall, P. L. (1990). The Focused Interview: a Manual of Problems and Procedures (2nd edn). Collier MacMillan.

McCabe, R., \& Priebe, S. (2004). Explanatory models of illness in schizophrenia: comparison of four ethnic groups. British Journal of Psychiatry, 185, 25-30.

McCulloch, A., Ryrie, I., Williamson, T., \& St John, T. (2005). Has the medical model a future? The Mental Health Review, 10, 7-15.

Morse, J., \& Field, P. (1996). Nursing Research: The Application of Qualitative Approaches. Chapman \& Hall.

Nazroo, J. Y. (1997). Ethnicity and Mental Health: Findings from a National Community Survey. Policy Studies Institute.

NICE (2014). Psychosis and Schizophrenia in Adults: Treatment and Management. NICE Clinical Guideline 178. London, UK: NICE. Available at: http://www.nice.org.uk/guidance/cg178/evidence/cg179-psychosis-and-schizophrenia-in-adultsfull-guidance 3 
NICE (2009). Schizophrenia: Core Interventions in the Treatment and Management of Schizophrenia in Adults in Primary and Secondary Care. NICE Clinical Guideline 82. London, UK: NICE.

NHS Digital (2017). Improving Access to Psychological Therapies (IAPT). Available at: https://digital.nhs.uk/data-andinformation/data-collections-and-data-sets/data-sets/improving-access-to-psychological-therapies-data-set-reports/improvingaccess-to-psychological-therapies-data-set-reports

Office for National Statistics (2001). Census of population. Available at: https://www.neighbourhood.statistics.gov.uk/

Office for National Statistics (2011). Ethnicity and National Identity in England and Wales: 2011. Available at: https://www. ons.gov.uk/peoplepopulationandcommunity/culturalidentity/ethnicity/articles/ethnicityandnationalidentityinenglandandwales/ 2012-12-11

Parkman, S., Davis, D., Leese, M., Phelan, M., \& Thornicroft, G. (1997). Ethnic differences in satisfaction with mental health services among representative people with psychosis in South London: PriSM Study 4. British Journal of Psychiatry, 171, 260-264.

Patel, S. (2016). An exploration of South Asian clients' experiences of therapist self-disclosure in individual therapy. Doctoral dissertation, City University of London.

Patel, V., \& Andrade, C. (2003). Pharmacological treatment of severe psychiatric disorders in the developing world: lessons from India. CNS Drugs, 17, 1071-1080.

Patterson, C. H. (1985). The Therapeutic Relationship: Foundations for an Eclectic Psychotherapy. Thomson Brooks/Cole Publishing Co.

Patterson, C. H., \& Hidore, S. (1997). Successful Psychotherapy: A Loving, Caring Relationship, pp. 111-113. Jason-Aronson.

Phiri, P. (2012). Adapting cognitive behaviour therapy for psychosis for black and minority ethnic communities. Doctoral thesis, University of Southampton, Faculty of Medicine.

Pinto, R., Ashworth, M., \& Jones, R. (2008). Schizophrenia in Black Caribbeans in the UK: an exploration of underlying causes of the high incidence rate. British Journal of General Practice, 58, 429-434.

Pinto-Coelho, K. G., Hill, C. E., \& Kivlighan Jr, D. M. (2016). Therapist self-disclosure in psychodynamic psychotherapy: a mixed methods investigation. Counselling Psychology Quarterly, 29, 29-52.

Polit, F., \& Hungler, P. (1999). Nursing Research: Principles and Methods (6th edn). Lippincott.

Rack, P. (1992). Race, Culture, and Mental Disorder. London, UK: Tavistock Publications.

Rathod, S., Kingdon, D., Smith, P., \& Turkington, D. (2005). Insight into schizophrenia: the effects of cognitive behavioural therapy on the components of insight and association with sociodemographics - data on a previously published randomised controlled trial. Schizophrenia Research, 74, 211-219.

Rathod, S., Naeem, F., Phiri, P., \& Kingdon, D. (2008). Expansion of psychological therapies. British Journal of Psychiatry, 193, 256.

Rathod, S., Kingdon, D., Phiri, P., \& Gobbi, M. (2010). Developing culturally sensitive cognitive behaviour therapy for psychosis for ethnic minority patients by exploration and incorporation of service users and health professionals views and opinions. Behavioural and Cognitive Psychotherapies, 38, 511-533

Rathod, S., Phiri, P., Harris, S., Underwood, C., Thagadur, M., Padmanabi, U., \& Kingdon, D. (2013). Cognitive behaviour therapy for psychosis can be adapted for minority ethnic groups: a randomised controlled trial. Schizophrenia Research, 143, 319-326.

Rathod, S.,Gega, L., Degnan, A., Pikard, J., Khan, T., Hussain, N., Munshi, T., \& Naeem, F. (2017). The Current Status of Culturally Adapted Mental Health Interventions: A Practice-Focused Review of Meta - Analyses. Neuropsychiatric Disease and Treatment. Dove Medical Press.

Raleigh, V. S. (2000). Mental health in black and ethnic minorities: an epidemiological perspective. In C. Kaye, and T. Lingiah (eds), Race, Culture and Ethnicity in Secure Psychiatric Practice. London and Philadelphia, Jessica Kingsley Publishers.

Richie, J., \& Lewis, J. (2003). Qualitative Research Practice: A Guide for Social Science Students and Researchers. Los Angeles: Sage Publications.

Rowan, J. (2008). A triumph for the humanistic approach: a customer's book review of Dimaggio et al. (2007). Psychotherapy of Personality Disorders: States of Mind and Interpersonal Cycles. Routledge. Available at: https://www.amazon.co.uk/ review/R13UNCHIN99Z (accessed 5 May 2009).

Sharpley, M., Hutchinson, G., Murray, R.M., \& McKenzie, K. (2001). 'Understanding the excess of psychosis among African-Caribbean population in England': review of current hypothesis. British Journal of Psychiatry, 178, 60-68.

Simone, D. H., McCarthy, P., \& Skay, C. (1998). An investigation of client and counsellor variables that influence likelihood of counsellor self-disclosure. Journal of Counselling and Development, 76, 174-182.

Simons, L., Lathlean, J., \& Squire, C. (2008). Shifting the focus: sequential methods of analysis with qualitative data. Qualitative Health Research, 18, 120-132.

Smith, J., Flowers, P., \& Larkin, M. (2009). Interpretative Phenomenological Analysis: Theory, Method and Research. London: Sage Publications. 
Specialist Library for Ethnicity and Health (SLEH) (2009). Schizophrenia and ethnic minorities in Britain: an urgent need to improve the evidence base. Published by SLEH. Available at: = http://www.library.nhs.uk/Ethnicity/ViewResource.aspx? resID $=282996$ andtabID $=290$ (accessed on 17 June 2009).

Spradley, J. P. (1980). Participant Observation. Fort Worth: Holt, Rinehart, and Winston.

Stewart, D. W., \& Shamdasani, P. N. (1990). Focus Groups: Theory and Practice. Sage Publications.

Strong, R. S. (1978). Social psychological approach to psychotherapy research. In Garfied L. \& Bergin A.E. (eds), Handbook of Psychotherapy and Behaviour Change (2nd edn), pp. 101-136. Wiley.

Sue, D. W., \& Sue, D. (2003). Counselling the Culturally Diverse: Theory and Practice (4th edn). Wiley.

Tseng, W.-S., Chang, S. C., \& Nishjzono, M. (2005). Asian Culture and Psychotherapy: Implications for East and West. USA, University of Hawaii Press.

Velthorst, E., Koeter, M., van der Gaag, M., Nieman, D.H., Felt, A.K., Smit, F. et al. (2015). Adapted cognitive behavioural therapy required for targeting negative symptoms in schizophrenia: meta-analysis and meta-regression. Psychological Medicine, 45, 453-465. doi.org/10.1017/S0033291714001147

Willig, C. (2008). Introducing Qualitative Research in Psychology: Adventures in Theory and Method (2nd edn). Maidenhead: McGraw-Hill Open University Press.

Wykes, T., Steel, C., Everitt, B., \& Tarrier, N. (2008). Cognitive behaviour therapy for schizophrenia: effect sizes, clinical models, and methodological rigor. Schizophrenia Bulletin, 34, 523-537.

Zimmermann, G., Favrod, J., Trieu, V. H., \& Pomini, V. (2005). The effect of cognitive behavioural treatment on the positive symptoms of schizophrenia spectrum disorders: a meta-analysis. Schizophrenia Research, 77, 1-9.

\section{Appendix 1}

\section{Inclusion criteria}

Inclusion criteria included the participants being:

- From a defined ethnic minority group with a diagnosis of schizophrenia/schizo-affective disorder/delusional disorder or a lay member from the ethnic minority group or MHP or CBT therapist.

- Willing to participate in the interview and have notes made and/or be tape recorded.

- Had capacity to consent and understand the interview.

\section{Exclusion criteria}

Exclusion criteria included:

- Severe illness that affected mental capacity or markedly affected their ability to participate in interview, e.g. very thought disordered or distressed by symptoms.

- Not agreeing to consent.

- Those patients who, in the opinion of the key worker/care coordinator, would become distressed by the interview.

- Patient identified that they are not of the stated ethnic group.

- Non-English speaking, because of the difficulty of conducting the interviews through interpreters.

Cite this article: Phiri P, Rathod S, Gobbi M, Carr H, and Kingdon D. Culture and therapist self-disclosure. The Cognitive Behaviour Therapist. https://doi.org/10.1017/S1754470X19000102 